\title{
Caso raro de anquilose da ATM oriunda de radioterapia: revisão sistemática de
}

\section{diferentes etiologias}

Rare case of TMJ ankyloses arising from radiotherapy: systematic review of different etiologies
Caso raro de anquilosis de la ATM por radioterapia: revisión sistemática de diferentes etiologías

Recebido: 24/08/2021 | Revisado: 02/09/2021 | Aceito: 07/09/2021 | Publicado: 09/09/2021

\author{
Bruno da Silva Mesquita \\ ORCID: https://orcid.org/0000-0002-2824-4088 \\ Universidade de Pernambuco, Brasil \\ E-mail: brunomesquitajpa@hotmail.com \\ Patrícia Verónica Aulestia Viera \\ ORCID: https://orcid.org/0000-0002-3860-2360 \\ Universidade de Pernambuco, Brasil \\ E-mail: paty98@usp.br \\ Ana Cláudia Amorim Gomes Dourado \\ ORCID: https://orcid.org/0000-0001-7731-7135 \\ Universidade de Pernambuco, Brasil \\ E-mail: anacagomes@uol.com.br \\ Emanuel Savio de Souza Andrade \\ ORCID: https://orcid.org/0000-0003-2165-4217 \\ Universidade de Pernambuco, Brasil \\ E-mail: manosavio@bol.com.br \\ Francisco Orlando Giraldi Neto \\ ORCID: https://orcid.org/0000-0003-0722-1140 \\ Hospital Municipal de Campo Limpo, São Paulo, Brasil \\ E-mail: giraldineto@bol.com.br \\ Ulisses Estevam Alves Neto \\ ORCID: https://orcid.org/0000-0001-7947-8530 \\ Faculdade de Enfermagem Nova Esperança, Brasil \\ E-mail: ulissesestevam21@gmail.com \\ Douglas da Cunha Vieira \\ ORCID: https://orcid.org/0000-0003-3710-6987 \\ Faculdade de Enfermagem Nova Esperança, Brasil \\ E-mail: douglascunha999@gmail.com
}

\begin{abstract}
Resumo
A anquilose da articulação temporomandibular ainda é um assunto em evidência na literatura sendo foco de várias divergências de opiniões principalmente no que se refere à etiologia e tratamento. Com base nisso, esse estudo tem como objetivo apresentar uma revisão sistemática sobre o tema ilustrando-o através de um relato caso clínico de anquilose bilateral de ATM que ocorreu como sequela de radioterapia utilizada no tratamento de um linfoepitelioma de nasofaringe. No caso relatado a anquilose bilateral da ATM se deu de forma progressiva por 20 anos. Essa condição foi tratada com remoção cirúrgica da anquilose e instalação de prótese customizada bilateral. Na revisão sistemática da literatura a etiologia mais referida foi o trauma, representando a grande maioria com 1185 casos, seguido de 110 casos de infecção, 1 de hiperplasia do processo coronoide da mandíbula, 102 idiopáticas, 17 congênitas, 10 problemas sistêmicos (como osteoartrite e espondilite) e 18 por cirurgia prévia. A radioterapia como fator etiológico para as anquiloses de ATM deve ser levada em conta sendo assim necessário a realização de estudos que sugiram protocolos de prevenção específicos.
\end{abstract}

Palavras-chave: Anquilose; Transtornos da articulação temporomandibular; Carcinoma.

\begin{abstract}
Temporomandibular joint ankylosis is still a subject in evidence in the literature, being the focus of several divergences of opinion, mainly regarding etiology and treatment. Based on this, this study aims to present a systematic review on the subject illustrated through a clinical case report of bilateral TMJ ankylosis that occurred as a sequela of radiotherapy used in the treatment of an auditory tube lymphoepithelioma. In the reported case, bilateral ankylosis of the TMJ progressively occurred for 20 years. This condition was treated with surgical removal of ankylosis and installation of bilateral custom prosthesis. In the systematic literature review, the most commonly referred etiology was trauma, representing the vast majority with 1185 cases, followed by 110 cases of infection, 1 of jaw choronoid process hyperplasia, 102 idiopathic, 17 congenital, 10 systemic problems (such as osteoarthritis and
\end{abstract}


spondylitis) and 18 by previous surgery. Radiotherapy as an etiologic factor for TMJ ankylosis should be taken into account and studies that suggest specific prevention protocols should be performed.

Keywords: Ankylosis; Temporomandibular joint disorders; Carcinoma.

\section{Resumen}

La anquilosis de la articulación temporomandibular sigue siendo un tema en evidencia en la literatura, siendo el foco de varias divergencias de opinión, especialmente en lo que respecta a su etiología y tratamiento. En base a esto, este estudio tiene como objetivo presentar una revisión sistemática sobre el tema, ilustrándolo a través de un reporte de caso clínico de anquilosis bilateral de ATM que ocurrió como secuela de la radioterapia utilizada en el tratamiento de un linfoepitelioma nasofaríngeo. En el caso que se informa aquí, la anquilosis bilateral de la ATM se produjo de forma progresiva durante 20 años. Esta condición se trató con la extirpación quirúrgica de la anquilosis y la colocación de una prótesis bilateral personalizada. En la revisión sistemática de la literatura, la etiología más reportada fue el trauma, representando la gran mayoría con 1185 casos, seguido de 110 casos de infección, 1 de hiperplasia de la apófisis coronoides de la mandíbula, 102 idiopática, 17 congénita, 10 problemas sistémicos (como artrosis y espondilitis) y 18 por cirugía previa. Se debe tener en cuenta la radioterapia como factor etiológico de la anquilosis de la ATM, por lo que es necesario realizar estudios que sugieran protocolos de prevención específicos.

Palabras clave: Anquilosis; Trastornos de la articulación temporomandibular; Carcinoma.

\section{Introdução}

A anquilose da articulação temporomandibular ainda é um assunto em evidência na literatura sendo foco de várias divergências de opiniões principalmente no que se refere à etiologia e tratamento. Patologias na ATM ou na região de cabeça e pescoço podem estar associadas com a anquilose e entre elas pode ser citado o linfoepitelioma, tipo raro de carcinoma relatado pela primeira vez em nasofaringe, mas sendo também descrito em região de glândulas salivares, timo, laringe, pulmão, colo uterino, pele, esôfago, estômago, cólon, reto e mais comumente em bexiga. As repercussões clínicas, sinais e sintomas, vão depender da localização da lesão. Em região de cabeça e pescoço são mais comuns os relatos de infecção tubária por obstrução do óstio da tuba na nasofaringe, obstrução nasal, epistaxe, dor facial, zumbido, cefaleia ou até parestesias, disestesias e paralisias por acometimento dos nervos cranianos. O tratamento pode ser radioterápico ou cirúrgico/radioterápico com a sua dose e intensidade a depender da localização, extensão e estágio da doença. Na literatura, são citadas algumas consequências pós radioterapia como trismo, xerostomia, osteorradionecrose, mucosite, disfagia, candidíase, otite e insuficiência velofaringea (Díaz, Esteban, Jesús \& Ace, 2018).

O trismo geralmente encontra-se presente entre 5 e $17 \%$ da pós-radioterapia e esse quadro pode estar relacionado com uma anquilose da articulação temporomandibular (AATM), que tanto pode ser causada por trauma dos côndilos mandibulares como estar relacionado a outros fatores locais a exemplo da infecção ou inflamação (Wu \& Lam, 2015). Essa condição pode ser resultante dos efeitos indesejados da radiação gerada na radioterapia utilizada no tratamento do linfoepitelioma que pode causar necrose, trombose de pequenos vasos e alterações nucleares pois a radiação age sobre o DNA celular ocasionando alterações importantes no comportamento e capacidade reprodutiva da célula e cominando na sua morte mitótica (Chatellier, Caillot, Diep, Be \& Veyssie, 2016).

Levando-se em consideração a metodologia adotada na construção desse trabalho percebe-se que até junho de 2019 o linfoepitelioma de nasofaringe ainda não foi relatado na literatura, bem como nenhuma sequela a longo prazo de anquilose da articulação temporomandibular, associada ao tratamento por radioterapia foi citada. Com base no pressuposto esse artigo tem como objetivo apresentar um estudo de caso clínico de anquilose bilateral de ATM que ocorreu como sequela de radioterapia utilizada no tratamento de linfoepitelioma de nasofaringe associado a uma revisão integrativa sobre tema.

\section{Metodologia}

Esta revisão sistemática foi registrada prospectivamente no PROSPERO (CRD42019120278) e seguiu a declaração PRISMA. É um estudo intervencional descritivo e qualitativo de relato de caso o qual possui a autorização do paciente por 
meio do Termo de Consentimento Livre e Esclarecido (TCLE), elaborado em linguagem acessível à compreensão do paciente relatado, evidenciando o destino das informações coletadas do paciente, assim como os benefícios e riscos associados à sua participação. Também foi feito uma pesquisa específica, foi adotada como base de dados principal a Pubmed na qual em 09 de novembro de 2019 utilizou-se os termos" (((ankylosis AND temporo-mandibular joint)) OR (ankylosis AND temporomandibular joint)) OR (ankylosis AND mandibular condyle)) OR (ankylosis AND etiology)", como metodologia de busca e foram encontrados 12923 artigos, Buscando atingir os níveis mais altos de evidência científica foram excluídos da análise os artigos de relatos de casos e revisões de literatura, chegando-se então em um total de 429 estudos selecionados. Também foram excluídos os artigos que não faziam menções às etiologias e aqueles nos quais não foi possível o acesso ao texto completo, sendo assim incluídos 9 artigos para revisão integrativa. Com os mesmos princípios também foi realizada uma busca alternativa na literatura cinza pela qual foram adicionados mais 3 artigos (Figura 1).

Figura 1: fluxograma da revisão integrativa.

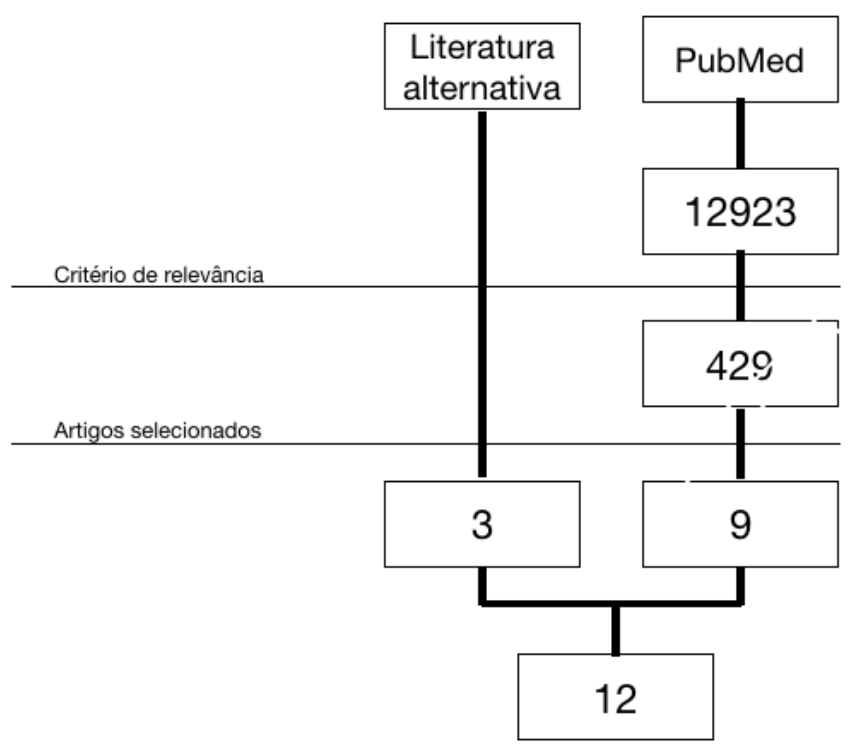

Fonte: Autores.

Dos 12 estudos selecionados 2 eram estudos retrospectivos, 2 observacionais, 1 série de casos, 1 revisão sistemática e 6 ensaios clínicos sendo 4 randomizados totalizando 1443 pacientes, desses 768 eram do sexo masculino e 646 do feminino com idade média de 18 anos.

A etiologia mais referida foi o trauma, representando a grande maioria com 1185 casos, seguido de 110 casos de infecção, 1 de hiperplasia do processo coronoide da mandíbula, 102 idiopáticas, 17 congênitas, 10 problemas sistêmicos (como osteoartrite e espondilite) e 18 por cirurgia prévia. 
Tabela 1: Estatística descritiva dos estudos incluídos.

\begin{tabular}{|c|c|c|c|c|c|c|c|c|c|c|c|c|c|c|c|c|c|c|}
\hline \multirow{2}{*}{ ID } & \multirow{2}{*}{ AUTOR } & \multirow{2}{*}{ TIPO DE ESTUDO } & \multirow{2}{*}{ ANO } & \multirow{2}{*}{$\mathbf{N}$} & \multirow{2}{*}{ IM } & \multicolumn{2}{|c|}{ SEXO } & \multicolumn{7}{|c|}{ ETIOLOGIA } & \multicolumn{4}{|c|}{ TRATAMENTO } \\
\hline & & & & & & M & $\mathbf{F}$ & TR & INF & $\mathrm{HC}$ & IDI & CONG & PS & CP & AG & AGI & AR & Ninf \\
\hline 1 & SU-GWAN & Retrospectivo & 2001 & 7 & 28 & 4 & 3 & 6 & 1 & 0 & 0 & 0 & 0 & 0 & 7 & 0 & 0 & 0 \\
\hline 2 & ROO et al. & Revisão sistemática & 2015 & 1165 & 17,9 & 619 & 546 & 966 & 85 & 0 & 79 & 13 & 8 & 14 & 390 & 279 & 138 & 0 \\
\hline 3 & JAKHAR; GUPTA & Estudo clínico & 2013 & 90 & 14,5 & 60 & 30 & 82 & 0 & 0 & 8 & 0 & 0 & 0 & 0 & 0 & 0 & 90 \\
\hline 4 & YANG et al. & Ensaio clínico ramdomizado & 2011 & 16 & 26,7 & 7 & 9 & 16 & 0 & 0 & 0 & 0 & 0 & 0 & 0 & 0 & 16 & 0 \\
\hline 5 & LIU et al. & Ensaio clínico ramdomizado & 2005 & 48 & 20,2 & 29 & 19 & 38 & 7 & 0 & 3 & 0 & 0 & 0 & 0 & 48 & 0 & 0 \\
\hline 6 & DANDA; RAMKUMAR & Ensaio clínico & 2009 & 16 & 10 & 9 & 7 & 13 & 2 & 0 & 1 & 0 & 0 & 0 & 8 & 8 & 0 & 0 \\
\hline 7 & MEHROTRA & Ensaio clínico ramdomizado & 2008 & 17 & 6,5 & 8 & 9 & 12 & 2 & 0 & 3 & 0 & 0 & 0 & 0 & 17 & 0 & 0 \\
\hline 8 & SPINELLI & Ensaio clínico ramdomizado & 2017 & 19 & 24,6 & 10 & 9 & 13 & 0 & 0 & 3 & 0 & 2 & 1 & 0 & 19 & 0 & 0 \\
\hline 9 & ADEBAYO & Relato de série de casos & 2012 & 23 & 20 & 13 & 10 & 11 & 9 & 1 & 0 & 2 & 0 & 0 & 0 & 0 & 0 & 23 \\
\hline 10 & VASCONCELOS; PORTO; NOGUEIRA & Observacional & 2008 & 6 & 17,5 & 4 & 2 & 1 & 3 & 0 & 0 & 2 & 0 & 0 & 0 & 0 & 6 & 0 \\
\hline 11 & GENARO et al. & Observacional & 2011 & 7 & 9 & 5 & 2 & 4 & 0 & 0 & 3 & 0 & 0 & 0 & 0 & 0 & 0 & 7 \\
\hline 12 & GOLDENBERG & Retrospectivo & 2010 & 29 & 13,5 & $x x x$ & $x x x$ & 23 & 1 & 0 & 2 & 0 & 0 & 3 & 29 & 0 & 0 & 0 \\
\hline TOTAL & & & & 1443 & 17,7 & 768 & 646 & 1185 & 110 & 1 & 102 & 17 & 10 & 18 & 434 & 371 & 160 & 120 \\
\hline
\end{tabular}

ID=Identificação; N= Total de pacientes; IM= Idade Média; M=Masculino; F= Feminino; TR=Trauma; INF= Infecção; HC= Hiperplasia de coronoide; $\mathrm{IDI}=$ Idiopática; $\mathrm{CONG}=$ Congênita; $\mathrm{OS}=$ Problemas Sistêmicos; $\mathrm{CP}=\mathrm{Cirurgia}$ Prévia; $\mathrm{AG}=\mathrm{Artroplastias}$ em $\mathrm{GAP}$; AGI= Artroplastias em GAP Interposicional; AR= Artroplastias reconstrutiva; DESC = Desconhecida; Ninf= Não informado. Fonte: Autores.

Os Tratamentos citados foram 434 casos artroplastias em gap, 371 artroplastias interposicionais, 160 artroplastias reconstrutivas. Em 120 casos o tratamento não foi informado.

\section{Relato de Caso}

Paciente do sexo masculino, 55 anos, procurou o serviço de cirurgia e traumatologia buco-maxilo-facial relatando dificuldade de abertura bucal e referindo evolução de 20 anos com agravamento gradual do caso, após o término da última sessão de radioterapia para tratamento de um linfoepitelioma de nasofaringe.

Essa lesão foi diagnosticada através de biopsia por linfadenectomia profunda em conjunto com exame imunohistoquimico. As características macroscópicas encontradas foram: fragmento nodular medindo 1,3 x 1,3 x 0,8 cm, apresentando superfície granulosa sendo constituído por tecido esbranquiçado com consistência aumentada. Ao exame microscópico observou-se fragmentos nódulo linfático com arquitetura geral apresentando-se preservadas em áreas, hiperplasia linfoide e áreas de proliferação fibrosa com presença de vasos que exibiam parede espessa, em meio aos elementos linfoides da cortical notaram-se blocos sólidos de tamanhos variados constituídos por células epiteliais que apresentavam núcleos vesiculosos ou hipercorados com nucléolos evidentes e intenso infiltrado linfoplasmocitário.

Ao exame clínico, além de uma assimetria facial com laterognatismo à direita, observou-se limitação severa de abertura bucal com $5 \mathrm{~mm}$ de amplitude máxima e um overbite de $3 \mathrm{~mm}$ quando em máxima intercuspidação habitual. Como consequência à limitação de abertura bucal, o paciente referiu dificuldade na alimentação, fala e higiene oral, que refletia diretamente em seu convívio social e saúde oral.

Ao exame tomográfico, foi possível observar na região de côndilo da mandíbula bilateralmente, uma extensa massa hiperdensa que se estendia desde o colo do côndilo da mandíbula até região de osso temporal, onde se encontrava na altura da fossa articular uma linha hipodensa. Dessa forma estabeleceu-se a hipótese diagnóstica de anquilose fibro-óssea extracapsular completa bilateral (Figura 2). 
Figura 2: Tomografia computadorizada das ATMs.

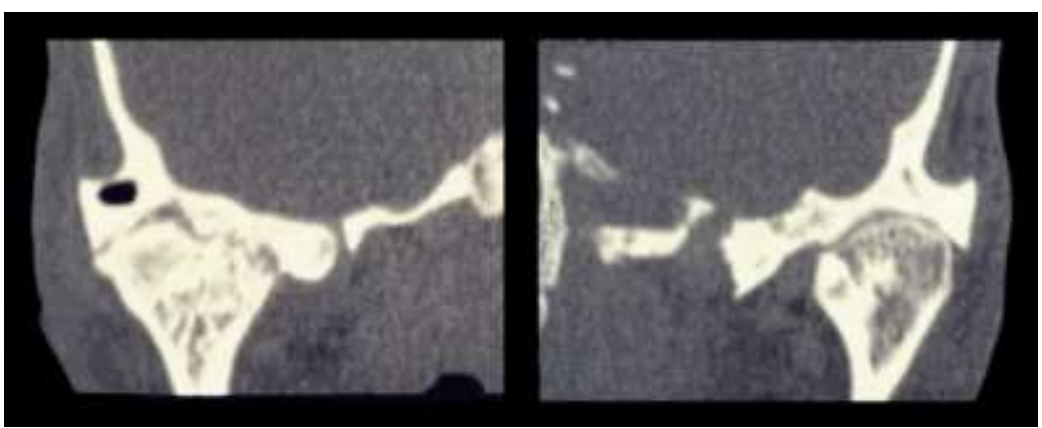

Fonte: Autores.

O tratamento proposto foi cirúrgico, sendo realizado a ressecção da massa anquilótica e coronoidectomia bilateral com reconstrução da articulação, utilizando-se próteses customizadas (Figura 3 a, b e c). Esse procedimento foi realizado sob anestesia geral, sendo a intubação nasotraqueal obtida com auxílio de fibroscopia e então, executado o acesso pré-auricular e submandibular bilateral. Deu-se início à ressecção cirúrgica e remoção do processo coronoide bilateral com a utilização de um piezoeléctrico.

Figura 3: Remoção da massa anquilótica. a) ATM direita, b) ATM esquerda e c) Processo coronoide hiperplasiado.

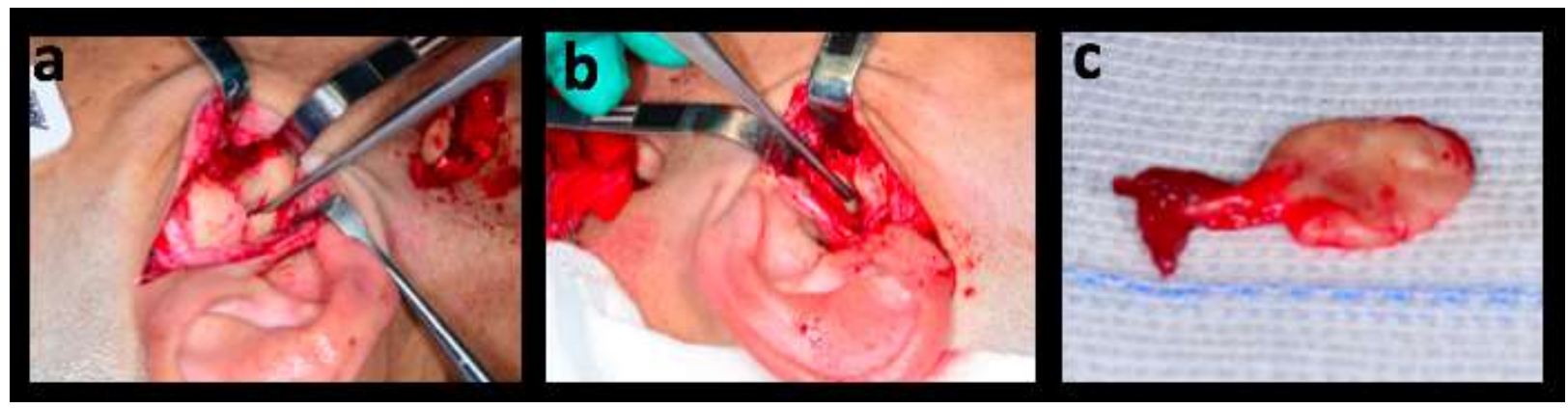

Fonte: Autores.

Em seguida, foi realizado o bloqueio maxilo-mandibular com auxílio de parafusos de bloqueio e instalada a prótese bilateral de côndilo (Figura 4 a, b e c), que se apresentou efetiva restabelecendo imediatamente a funcionalidade da articulação, confirmada no transcirúrgico com abertura bucal forçada máxima de 25mm (Figura 04 d). 
Figura 4: a) prótese customizada de ATM; b) Prótese customizada de ATM instalada do lado direito; c) Prótese de ATM instalada do lado esquerdo.

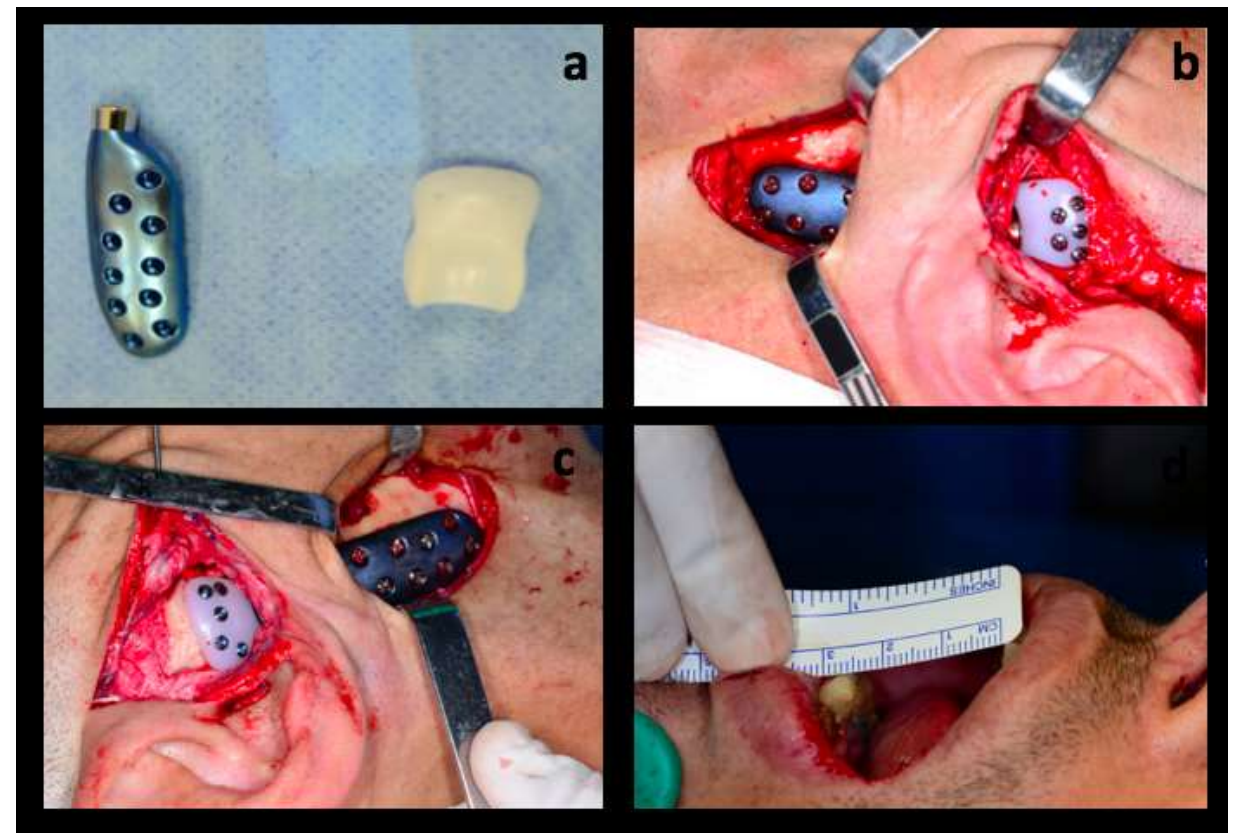

Fonte: Autores.

No acompanhamento pós-operatório, foi instituído o tratamento multidisciplinar com fisioterapia, fonoaudiologia e psicologia. Após 5 anos de acompanhamento, o paciente apresentava-se sem recidivas ou complicações e abertura bucal preservada com máxima de $30 \mathrm{~mm}$.

\section{Discussão}

O linfoepitelioma de nasofaringe é um câncer de localização rara e devido sua posição e relação anatômica com estruturas nobres o tratamento de escolha é radioterápico. No presente caso, foi utilizada uma radioterapia convencional bidimensional com uma dose de radiação variando entre 66 e 70 Gy, que foi efetiva contra o tumor. Porém, o paciente evoluiu com trismo mandibular progressivo após término da sua última sessão radioterápica. Algumas alternativas mais avançadas estão disponíveis na medicina oncológica, como a radioterapia de intensidade modulada e a terapia de arco modulado volumétrico, que oferecem a possibilidade de uma dose radioativa maior e mais concentrada no volume bruto do tumor, preservando estruturas vizinhas e consequentemente, reduzindo a probabilidade do aparecimento de complicações futuras (Eekers et al., 2018).

Sabe-se que o trismo é uma complicação tardia esperada após a realização de radioterapia em região de cabeça e pescoço. Essa condição surge rapidamente nos nove primeiros meses de pós radioterapia e depois disso progride de forma mais lenta chegando a uma diminuição de abertura bucal de $32 \%$ após quatro anos. Alterações como fibrose radioativa, diminuição gradual da vascularização e atrofia dos músculos articulares são citados na literatura como possíveis causas do trismo pós radioterapia. De fato, essas situações devem ser levadas em consideração. Porém, a associação desses fatores com efeitos colaterais causados diretamente nos tecidos locais da ATM, como a osteorradionecrose, podem juntamente com a negligencia do profissional e do paciente levar a um quadro de anquilose da articulação (Wu \& Lam, 2015).

O câncer é um diagnóstico que apresenta um grande impacto psicológico para paciente, o risco de morte e a busca pela possível cura ou maior sobrevida possível passa a ser o principal foco e objetivo para o doente e equipe de saúde. Isso é 
esperado até porque o desenvolvimento e evolução dos tratamentos disponíveis aumentam cada vez mais a sobrevida dos pacientes e com isso, as complicações começam a ser percebidas mais tardiamente, já em um quadro mais avançado. Um acompanhamento multidisciplinar adotado desde o diagnóstico até a fase de acompanhamento poderia reduzir essas sequelas e proporcionar uma melhor qualidade de vida ao paciente. Como exemplo, o caso aqui relatado que chegou ao serviço de cirurgia buco-maxilo-facial com quadro extremamente avançado de anquilose verdadeira que só poderia ser resolvido com intervenção cirúrgica. Contudo, um acompanhamento mais precoce com implementação de condutas conservadoras poderia ter evitado esse quadro (Ochoa, Casellas-grau, Vives \& Font, 2016).

Os principais objetivos do tratamento da anquilose visam restabelecer o movimento de articulação, incluindo a abertura bucal adequada, proporcionar uma oclusão funcional, reconstruir o conjunto articular e evitar a recorrência. Alguns estudos, citam o tratamento cirúrgico com ressecção da massa anquilótica em gap e proservação do caso com fisioterapia intensiva, a fim de não ocorrer recidiva e só posteriormente, realizar a reconstrução da articulação em um segundo momento cirúrgico. Porém, na maioria dos casos esse princípio é bem adequado para pacientes muito jovens que não tenham atingido a idade de maturação óssea e possuam um metabolismo acelerado com fator de crescimento ativo e que não tenham nenhuma doença sistêmica ou condição local que afete o processo de reparo tecidual (Farin, Canto, Gunckel, Alister, \& Uribe, 2017).

Em um estudo que comparou pacientes com complicações autoimunes que foram submetidos a reconstruções autógenas e alógenas da ATM percebeu-se que os melhores resultados de tratamento foram obtidos com a reconstrução total da articulação com próteses aloplásticas. Dessa forma, tendo em vista a história médica do paciente, idade e esperadas características específicas do local de interesse, optou-se para o caso reportado um tratamento cirúrgico com remoção da anquilose e reconstrução da articulação com prótese de ATM customizada e coronoidectomia bilateral em um único momento cirúrgico, evitando maior morbidade, como necessidade de sítios doadores de enxerto, reduzindo o tempo de tratamento e restabelecendo os parâmetros funcionais de maneira mais imediata (Mehra, Henry \& Nadershah, 2018).

\section{Considerações Finais}

Como conclusão citamos que mais importante do que a raridade da localização do tumor é o conhecimento de possíveis complicações decorrentes do tratamento radioterápico sendo de extrema importância para a equipe de saúde envolvida como também para o próprio paciente. Com base na disponibilidade atuais novas tecnologias alternativas mais efetivas e revisão nas doses radioterápicas necessárias para tratamento de tumores próximos à região de articulação temporomandibular devem ser estudadas de maneira multidisciplinar. A radioterapia como fator etiológico para as anquiloses das articulações temporo-mandibulares deve ser levada em conta sendo assim necessário a realização de estudos que sugiram protocolos de prevenção para esses casos.

\section{Referências}

Adebayo S., Aluko B., Ademola A., \& Ajike SO. (2012). Aetiology and presentation of ankylosis of the temporomandibular joint: report of 23 cases from Abuja, Nigeria.. 50, 80-4.

Chatellier A., Caillot A., Diep D., Be H \& Veyssie A. L. (2016). ankylose temporo-mandibulaire. 1-11.

Danda A. K. \& Ramkumar S. (2009). Comparison of Gap Arthroplasty With and Without a Temporalis Muscle Flap for the Treatment of Ankylosis. YJOMS . American Association of Oral and Maxillofacial Surgeons. 67(7):1425-31.

Díaz C., Esteban F., Jesús M., \& Ace F. (2018). Patologí a Lymphoepithelioma-like carcinoma of the large intestine : A case report and literature review. 51(1):18-22.

Eekers D. B. P., Roelofs E., Cubillos-mesías M., Niël C., Smeenk R. J., \& Hoeben A. (2018). Intensity-modulated proton therapy decreases dose to organs at risk in low-grade glioma patients : results of a multicentric in silico ROCOCO trial. Acta Oncol (Madr). Taylor \& Francis. 0(0):1-9.

Estrela, C. (2018). Metodologia Científica: Ciência, Ensino, Pesquisa. Editora Artes Médicas.

Farin R., Canto Ã. L., Gunckel R., Alister J. P., \& Uribe F. (2017).Temporomandibular Joint Ankylosis : Algorithm of Treatment.00(00):1-5. 
Research, Society and Development, v. 10, n. 11, e545101119811, 2021

(CC BY 4.0) | ISSN 2525-3409 | DOI: http://dx.doi.org/10.33448/rsd-v10i11.19811

Genaro K. F., Christinny D., Oliveira B. De., \& Passos F. (2011). Atividade muscuar da mastigação. (1).

GolDenber G. D. C., Alonso N. A., BaDotti R. B., \& Bastos D. G., (2010). Análise dos resultados do tratamento da anquilose da articulação temporomandibular com e sem distração mandibular. 13(2):69-72.

Jakhar S. K., \& Gupta M. A. K,. (2013). Preservation of condyle and disc in the surgical treatment of type III temporomandibular joint ankylosis : a long-term follow-up clinical study of 111 joints. Int J Oral Maxillofac Surg. International Association of Oral and Maxillofacial Surgery. 42(6):746-51.

Liu Y., Li J., Hu J., Zhu S., \& Luo E. (2005). Autogenous coronoid process pedicled on temporal muscle grafts for reconstruction of the mandible condylar in patients with temporomandibular joint ankylosis. YMOE. Elsevier Inc.109(2):203-10.

Mehra P., Henry C., \& Nadershah M.. T. M. J. (2018). Reconstruction in Patients with Autoimmune/Connective Tissue Disease. J Oral Maxillofac Surg. The American Association of Oral and Maxillofacial Surgeons.

Mehrotra D., Pradhan R., Mohammad S., \& Jaiswara C. (2008). Random control trial of dermis-fat graft and interposition of temporalis fascia in the management of temporomandibular ankylosis in children.. 46:521-6.

Ochoa C., Casellas-grau A., Vives J., \& Font A. (2016). Positive psychotherapy for distressed cancer survivors : Posttraumatic growth facilitation reduces posttraumatic stress. Int J Clin Heal Psychol. Asociación Española de Psicología Conductual.

Roo N. D., Doorne L. V., Troch A., Vermeersch H.., \& Brusselaers N. S. C. J. (2015). Cranio-Maxillo-Facial Sur. Elsevier Ltd.

Spinelli G., Valente D., Mannelli G., Raffaini M., \& Arcuri F. (2017). Surgical management of ankyloses of the temporomandibular joint by a piezoelectric device. J Cranio-Maxillo-Facial Surg. Elsevier Ltd.

Su-gwan K., (2001). Clinical paper : Treatment of temporomandibular joint ankylosis with temporalis muscle and fascia flap. 189-93.

Vasconcelos B. C. E., Porto G. G., \& Nogueira R. V. B. (2008). Anquilose da articulação têmporo-mandibular Temporo mandibular joint ankylosis.74(1):34-

Wu V. W. C., \& Lam Y. (2015). Radiation-induced temporo-mandibular joint disorder in post-radiotherapy nasopharyngeal carcinoma patients : assessment and treatment. $1-9$.

Yang X., Hu J., Yin G., Hu J., \& Luo E. (2011). Computer-assisted condylar reconstruction in bilateral ankylosis of the temporomandibular joint using autogenous coronoid process. Br J Oral Maxillofac Sur. British Association of Oral and Maxillofacial Surgeons. 49(8):612-7. 